\title{
On a possible approximation of discontinuous dynamical systems
}

\author{
Marius-F. Danca $^{\mathrm{a}, *}$, Steliana Codreanu ${ }^{\mathrm{b}}$ \\ ${ }^{a}$ Department of Mathematics, Spiru Haret College, 3400 Cluj-Napoca, Romania \\ ${ }^{\mathrm{b}}$ Department of Theoretical Physics, Babes-Bolyai University, 3400 Cluj-Napoca, Romania
}

Accepted 3 January 2001

\begin{abstract}
This work presents the arguments for the possibility of approximating some discontinuous dynamical systems with continuous or even smooth dynamical systems. The discontinuous differential equation, which describes the system, is transformed into a differential inclusion and then into a differential equation with continuous or smooth right-hand side. As an example a generalization of the equations governing Chua's circuit is presented. (c) 2001 Elsevier Science Ltd. All rights reserved.
\end{abstract}

\section{Introduction}

It is well known that many dynamical systems are modeled by autonomous ordinary differential equations with the underlying Cauchy problem, which can be written in the vector form

$$
\dot{x}(t)=f(x(t)), \quad x(0)=x_{0},
$$

where $f$ is an $n$-dimensional vector-valued, usually continuous, function $f: \Omega \subseteq \mathbf{R}^{n} \rightarrow \mathbf{R}^{n}, x_{0} \in \Omega$. If $f$ is a continuous function, then we call a solution of (1) a continuous differentiable vector-valued function $x(\cdot):[0, \infty) \rightarrow \mathbf{R}^{n}$ satisfying (1) for all $t \in[0, \infty)$. In this case, the Péano theorem assures the existence of this solution. Details about the existence and uniqueness of the solutions of the initial value problem (1) can be found, e.g., in [1].

Although the great majority of studied dynamical systems are modeled by initial value problems of the form (1), with $f$ a continuous function, there are many practical situations when $f$ is discontinuous, with respect to the state variable $x$ or/and to the time variable $t$. In the present paper we consider models with right-hand side discontinuous with respect to $x$, which are specific for a whole variety of applications, e.g., mechanical systems with dry friction, the Prager-Ishlinsky model in the theory of plasticity, electric circuits with small inductivities, systems with small inertia, dynamical systems with nondifferentiable potential, optimization problems with nonsmooth data, electrical networks with switches, oscillations in visco-elasticity, optimal control, etc. (see, e.g., [2,3] and references therein).

The existence and the uniqueness of the solutions in these cases is a delicate problem, as can be seen from the following example. Let us consider the equation [4]

$$
\dot{x}=1-2 \operatorname{sgn} x
$$

\footnotetext{
* Corresponding author.

E-mail address: marius.danca@aut.utcluj.ro (M.-F. Danca).
} 

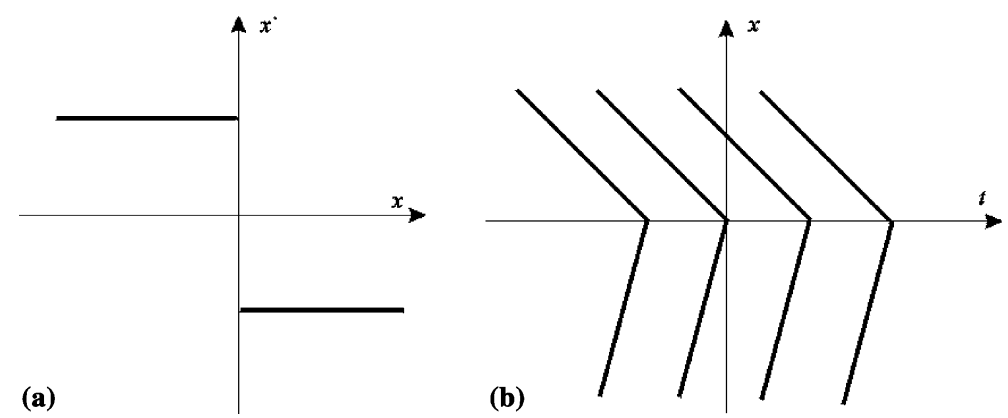

Fig. 1. (a) Function $f(x)=1-2 \operatorname{sgn} x$ represented in the state plane $(\dot{x}, x)$. (b) Solutions of the equation $\dot{x}=f(x)$.

where

$$
\operatorname{sgn} x= \begin{cases}-1 & \text { for } x<0 \\ 0 & \text { for } x=0 \\ +1 & \text { for } x>0\end{cases}
$$

and $f: \mathbf{R} \rightarrow \mathbf{R}$. Here $f(x)=1-2 \operatorname{sgn} x$ is a discontinuous function at the point $x=x^{*}=0$ (Fig. 1(a)). It is easy to see that the equation has different solutions for $x \neq 0$ :

$$
x(t)= \begin{cases}3 t+C_{1} & \text { for } x<0 \\ -t+C_{2} & \text { for } x>0\end{cases}
$$

where $C_{1}$ and $C_{2}$ are some constants (see Fig. 1(b)). We observe that increasing $t$, the solutions approach the line $x=0$, but the underlying function, $x(t)=0$, does not satisfy the equation since $\dot{x}(0)=0 \neq 1-2 \operatorname{sgn}(0)$.

It is obvious that the uniqueness, even the existence, in the usual sense, of the solutions of a discontinuous initial value problem, is a problem which needs special mathematical analysis. The background of discontinuous dynamical systems can be found in Filippov's book [4].

Our present work is an attempt to approximate systems modeled by discontinuous differential equations with continuous, even smooth ones. The algorithm we propose is the following: the equation discontinuous with respect to the state variable is transformed first into a differential inclusion and then into a differential equation with continuous (smooth) right-hand side. The obtained system can be qualitatively analyzed with the theory of continuous dynamical systems.

This algorithm is presented in Section 2, where some definitions and results are detailed in Appendix A. In Section 3, as an example, a generalization of equations governing Chua's circuits is discussed. Some conclusions follow in Section 4.

\section{The algorithm}

Let us consider the following autonomous dynamical system discontinuous with respect the state variable $x$ :

$$
\dot{x}(t)=f(x(t)), \quad x(0)=x_{0}, \quad t \in[0, \infty),
$$

where $f: \Omega \rightarrow \mathbf{R}^{n}$ is a vector-valued function discontinuous on a null set $M, \Omega$ being an open subset of $\mathbf{R}^{n}$. We consider that $f$ has bounded discontinuities, i.e., if $x^{*} \in M, f$ tends to finite (possible different) limits when its argument approaches $x^{*}$ (see Figs. 2(a) and 1(a), for $n=1$ ). We can consider without loss of generality that $M$ consists in a single point $x^{*}$, i.e. $M=\left\{x^{*}\right\}$. Let $\mathscr{F}$ be the class of these functions.

In order to find the assumptions on $f$ on which the discontinuous initial value problem (2) can be transformed into a continuous one, we will define a new function $F$, which, at the continuity points of $f$, coincides with $f$ and at the discontinuity point $x^{*}$, is chosen to be, for example, the convex hull of the function $f$ (Definition 1 in Appendix A). We note that other choices of $F$ are also possible $[4,7,8]$. 


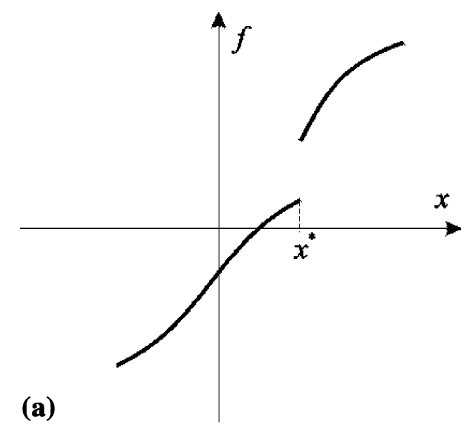

(a)

Fig. 2. (a) A function $f: \mathbf{R} \rightarrow \mathbf{R}$ discontinuous at $x=x^{*}$.

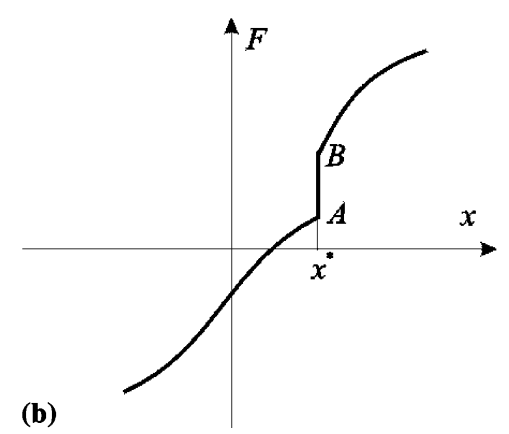

(b) The smallest convex hull $F$ for $f$.

It is obvious that the function $F$ is a vector set-valued function, defined on entire domain $\Omega, F: \Omega \Rightarrow \mathbf{R}^{n}$, with values into the set of all closed and convex subsets of $\mathbf{R}^{n}$, which associates to a discontinuity point $x=x^{*}$ a subset, $F\left(x^{*}\right)$, i.e., $F$ has several (even an infinite number of) values at the discontinuity point $x^{*}$.

As example, for the functions $f \in \mathscr{F}$ when $n=1$, the graph of $F$, Graph $(F)$ (see Definition 2 in Appendix A), is just the segment joining in $x^{*}$ the endpoints of $f$ (the segment $A B$, in Fig. 2(b)); when $n=2, F$ is a surface, as will be later illustrated in Section 3.

The above definition of $F$ is due to Filippov [4] and is called Filippov regularization (see Filippov regularization in Appendix A). Generally, the functions $f \in \mathscr{F}$ present a jump in $x^{*}$, due to the signum function. Then Filippov regularization gives us the sigmoid-like set-valued function, Sgn

$$
\operatorname{Sgn} x= \begin{cases}\{-1\} & \text { for } x<0 \\ {[-1,1]} & \text { for } x=0 \\ \{+1\} & \text { for } x>0\end{cases}
$$

Using Filippov regularization, the differential equation (2) is transformed into the following differential inclusion:

$$
\dot{x}(t) \in F(x(t)), \quad x(0)=x_{0} \text { for almost } t \in I,
$$

where $F: \Omega \Rightarrow \mathbf{R}^{n}$ is a set-valued function. The differential inclusion (3) reads as follows: for each value of $x \in \Omega$, the value of $\dot{x}$ belongs to the set of values $F(x)$, which equals $f(x)$ for $x \neq x^{*}$. Thus, we have embedded $f$ into a set-valued map $F$ which, as a set-valued function, has enough properties to have trajectories closely related to the trajectories of the original equations (2). Following this way, we can ignore possible misbehavior on sets of null measure (here the point $x^{*}$ ).

The differential inclusions (3) may have a unique or several solutions. By solution we mean here an absolutely continuous vector-valued function $x(\cdot):[0, \infty) \rightarrow \mathbf{R}^{n}$ (see Definition 3 in Appendix A) verifying (3) almost everywhere on $I$ (i.e., except the values of $t$ corresponding to $x^{*}$ ). An extension of Péano's existence theorem for differential inclusions exists [7,8]. We must note that, generally, the solutions are continuous but not differentiable (see Remark 1 in Appendix A).

Now, the next step is to find a so-called selection of the function $F$ (see Definition 4 in Appendix A), meaning an explicit single-valued function $g: \Omega \rightarrow \mathbf{R}^{n}$ which approximates $F$ in some neighborhood of Graph $(F)$. There are several possibilities for choosing such selections. One of the selection existence theorems is Cellina's theorem (see Theorem 1 in Appendix A) that assures the existence of continuous selections. An important remark is that all the set-valued functions obtained by Filippov regularization applied to functions $f \in \mathscr{F}$ verify the conditions required by the above theorem.

Let us apply Theorem 1 only on a closed neighborhood $U=\left[x^{*}-\varepsilon, x^{*}+\varepsilon\right]$ of the discontinuity point $x^{*}$. In this case we must choose the selection $g: U \rightarrow \mathbf{R}^{n}$ such that $g(x)=f(x)$, for any $x$ which belongs to the frontier of $U$. Moreover, if $f$ is smooth on $\Omega \backslash\left\{x^{*}\right\}$, we can choose $g$ to be smooth too. Hence we must impose the differentiability conditions to $g$ on the frontier of $U$. In Fig. 3 we illustrate a possible continuous differentiable selection for the set-valued function $F$ in the neighborhood $U$ of $x^{*}$, for $n=1$. 


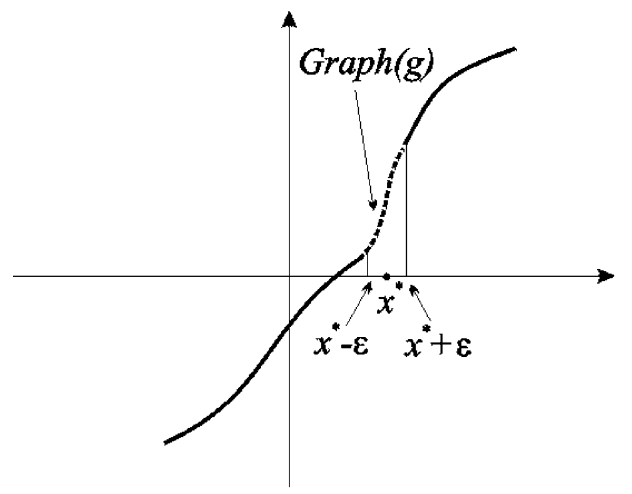

Fig. 3. A selection in a neighborhood of $\operatorname{Graph}(F)$ for $n=1$.

For physical reasons the width $\varepsilon$ of the neighborhood must be sufficiently small so that the motion of the physical system differs arbitrarily little from the solution of the approximated system. Finally we obtain a continuous (smooth) dynamical system

$$
\dot{x}(t)=h(x(t)),
$$

where

$$
h(x)= \begin{cases}f(x) & \text { for } x \notin U, \\ g(x) & \text { for } x \in U,\end{cases}
$$

is a vector-valued function from $\bar{\Omega}$ (the closure of $\Omega$ ) into $\mathbf{R}^{n}$.

Thus, we proved that, following the above steps, we can transform the discontinuous system (2) into a continuous (smooth) one (4). This system can be now analyzed with the classical tools of continuous dynamical systems theory.

\section{Example}

We choose, in order to exemplify the above transformation, the following autonomous system of discontinuous differential equations which is one of the generalizations of the equations governing Chua's circuit proposed in [9], which has no global classical solution on $[0, \infty)$ :

$$
\left(\begin{array}{c}
\dot{x}_{1} \\
\dot{x}_{2} \\
\dot{x}_{3}
\end{array}\right)=\left[\begin{array}{ccc}
-\alpha(b+1) & \alpha & 0 \\
1 & -1 & 1 \\
0 & -\beta & 0
\end{array}\right]\left(\begin{array}{c}
x_{1}-k \operatorname{sgn} x_{1} \\
x_{2} \\
x_{3}+k \operatorname{sgn} x_{1}
\end{array}\right) .
$$

For numerical reasons we consider, like in Brown's work [9] $k=1.5,-\alpha(b+1)=-18 / 7, b=-5 / 7, \alpha=9$ and $\beta=15$. Thus (5) becomes

$$
\begin{aligned}
& \dot{x}_{1}=-2.571 x_{1}+9 x_{2}+3.857 \operatorname{sgn} x_{1}, \\
& \dot{x}_{2}=x_{1}-x_{2}+x_{3}, \\
& \dot{x}_{3}=-15 x_{2} .
\end{aligned}
$$

The system (6) is discontinuous at $x^{*}=\left(0, x_{2}, x_{3}\right)$ due to the first component of the right-hand side $f_{1}\left(x_{1}, x_{2}\right)=-2.571 x_{1}+9 x_{2}+3.857 \operatorname{sgn} x_{1}$. Hence the discontinuity surface of the system has the equation $x_{1}=0$ (see Figs. 4(a), (b)). 

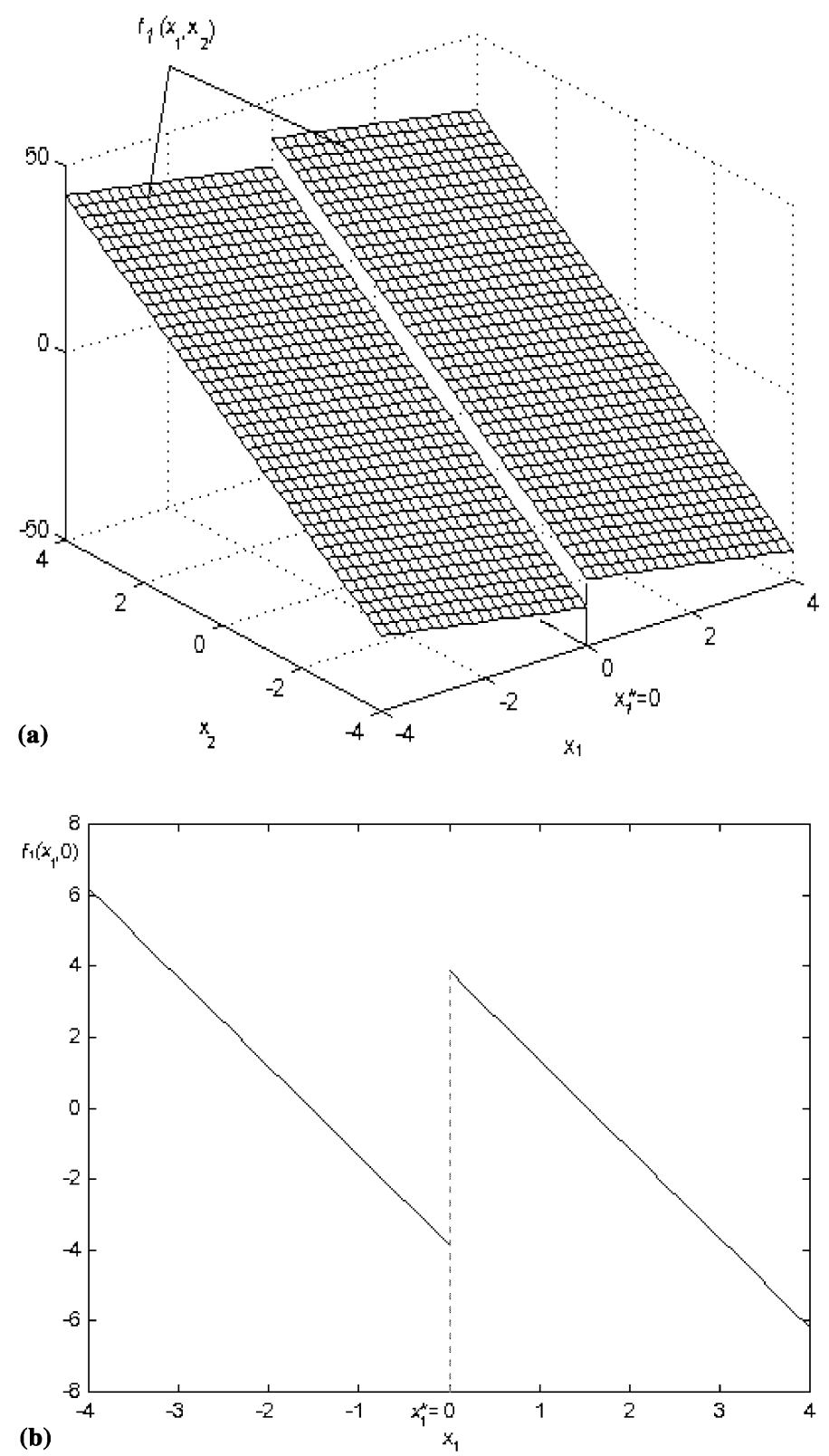

Fig. 4. (a) The surface $f_{1}\left(x_{1}, x_{2}\right)$ of the first component function of the right-hand side of (6). (b) An intersection of $f_{1}\left(x_{1}, x_{2}\right)$ with the plane $x_{2}=0$.

The set-valued function $F$ is obtained using the set-valued function Sgn. Therefore the system (6) becomes

$$
\begin{aligned}
& \dot{x}_{1} \in-2.571 x_{1}+9 x_{2}+3.857 \operatorname{Sgn} x_{1}, \\
& \dot{x}_{2}=x_{1}-x_{2}+x_{3}, \\
& \dot{x}_{3}=-15 x_{2},
\end{aligned}
$$

which is a differential inclusion. Hence $f_{1}$ is transformed into the set-valued function $F_{1}$ (see Fig. 5):

$$
F_{1}\left(x_{1}, x_{2}\right)=-2.571 x_{1}+9 x_{2}+3.857 \operatorname{Sgn} x_{1} .
$$

The convex hull for $x_{1}=0$ is just the shaded surface. 


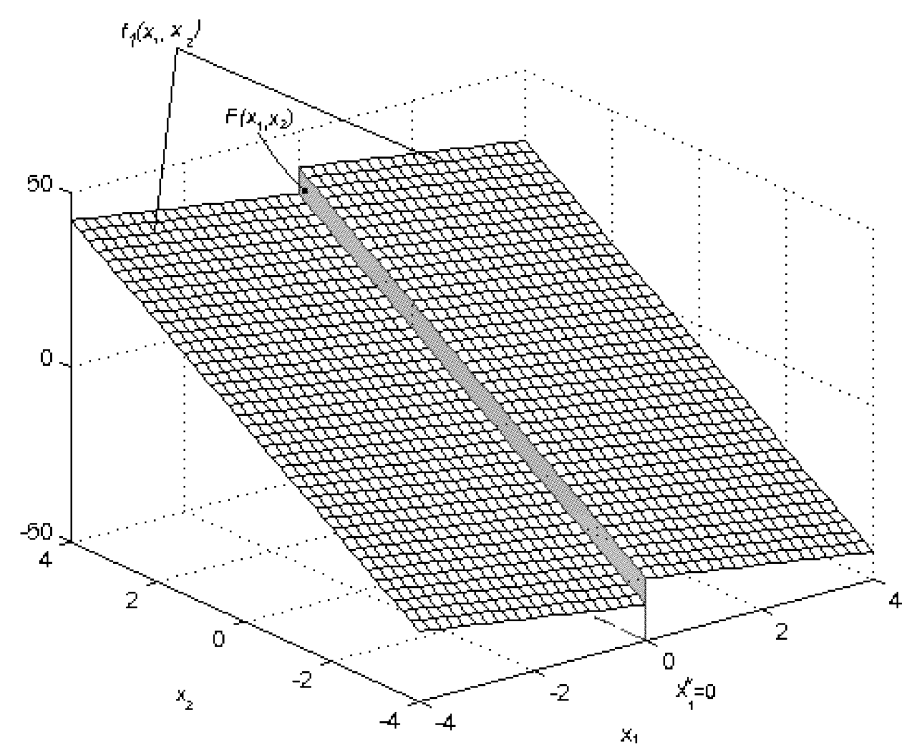

Fig. 5. The set-valued function $F_{1}$ corresponding to the differential inclusion (7).

Next, applying Theorem 1 in a neighborhood of radius $\varepsilon>0$ of the plane $x_{1}=0$, we can replace the setvalued function $F_{1}$ with a smooth function $g$ (here a cubic surface),

$$
g\left(x_{1}, x_{2}\right)=a x_{1}^{3}+b x_{1}^{2}+c x_{1}+d+9 x_{2},
$$

where $a, b, c$, and $d$ have to be determined from the continuity and differentiability conditions in $x_{1}^{*} \pm \varepsilon= \pm \varepsilon$ (see Figs. 6(a), (b)).

Thus, we obtain the following continuous smooth system, which is an approximation of the initial discontinuous system (5):

$$
\begin{aligned}
& \dot{x}_{1}=h\left(x_{1}, x_{2}\right)=g\left(x_{1}, x_{2}\right)-2.571 x_{1}, \\
& \dot{x}_{2}=x_{1}-x_{2}+x_{3}, \\
& \dot{x}_{3}=-15 x_{2} .
\end{aligned}
$$

The continuous system (8) is numerically integrated using a standard Runge-Kutta scheme. Two trajectories, for $\varepsilon=0.05$ and $\varepsilon=0.1$, which are chaotic for $\beta=15$ (compare [9]) are illustrated in Figs. 7(a) and (b). In Fig. 7(c) a trajectory of differential inclusion (7) obtained with the explicit Euler method for differential inclusions (see [2,3]) is plotted. More accurate results can be obtained using multistep numerical methods with high consistence order as Runge-Kutta (compare $[5,6]$ ).

From these figures, the influence of $\varepsilon$ can be deduced. Also it can be seen that the motion for the differential equations (8) and differential inclusion (7) are slightly different. However, by choosing $\varepsilon$ to be small enough, the algorithm can be used successfully.

In Fig. 8 a three-dimensional trajectory is plotted.

\section{Conclusions}

A discontinuous dynamical system modeled by the initial value problem (2), with $f \in \mathscr{F}$, can be approximated with a continuous (smooth) one, by applying the algorithm shown in Fig. 9.

We note that, the validity of this approximation being proved, for practical problems, the discontinuous system can be directly approximated with a continuous one (the dashed path in Fig. 9). 

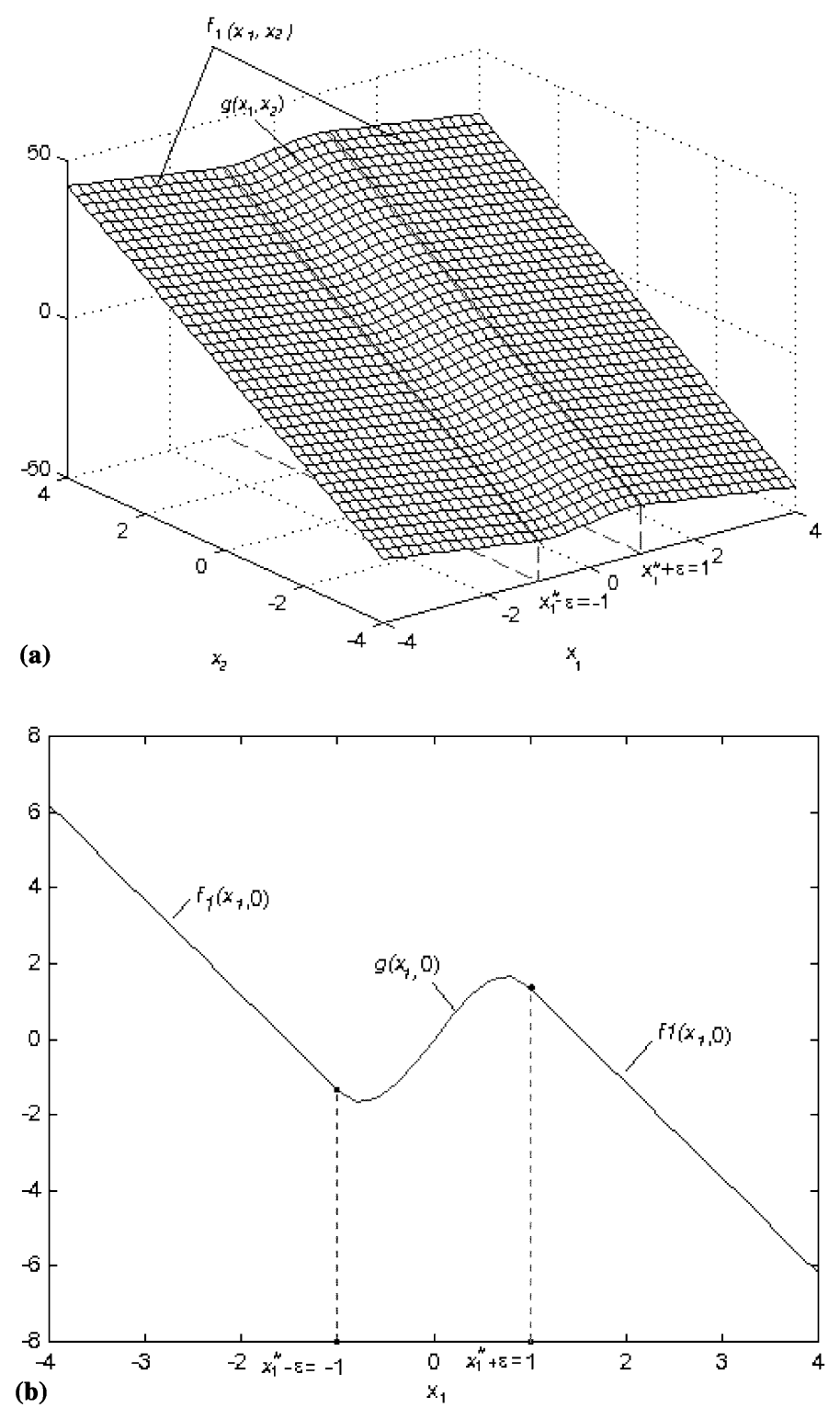

Fig. 6. (a) The selection $g$ of the set-valued function $F_{1}$. (b) The intersection of the graph of selection $g$ with the plane $x_{2}=0$.

We used this algorithm in the case of autonomous discontinuous systems, but it is also valid for nonautonomous systems which can be easily transformed into autonomous systems.

The main result is in fact a way to avoid mathematical complications when a discontinuous system has to be analytically or numerically analyzed.

The general case of the set $M$ of discontinuity points can be treated in the same way as that of the single point $x^{*}$.

The problem approached in this paper is more complex and can formulate a hypothesis that this procedure can be applied in the case of chaotic systems only for certain ranges of the control parameter. Hence "false" chaotic (strange) attractors could be obtained (see [10]). Nevertheless, this approach confirms the practical usefulness of the above continuous approximation, especially for further investigations. 
(a)
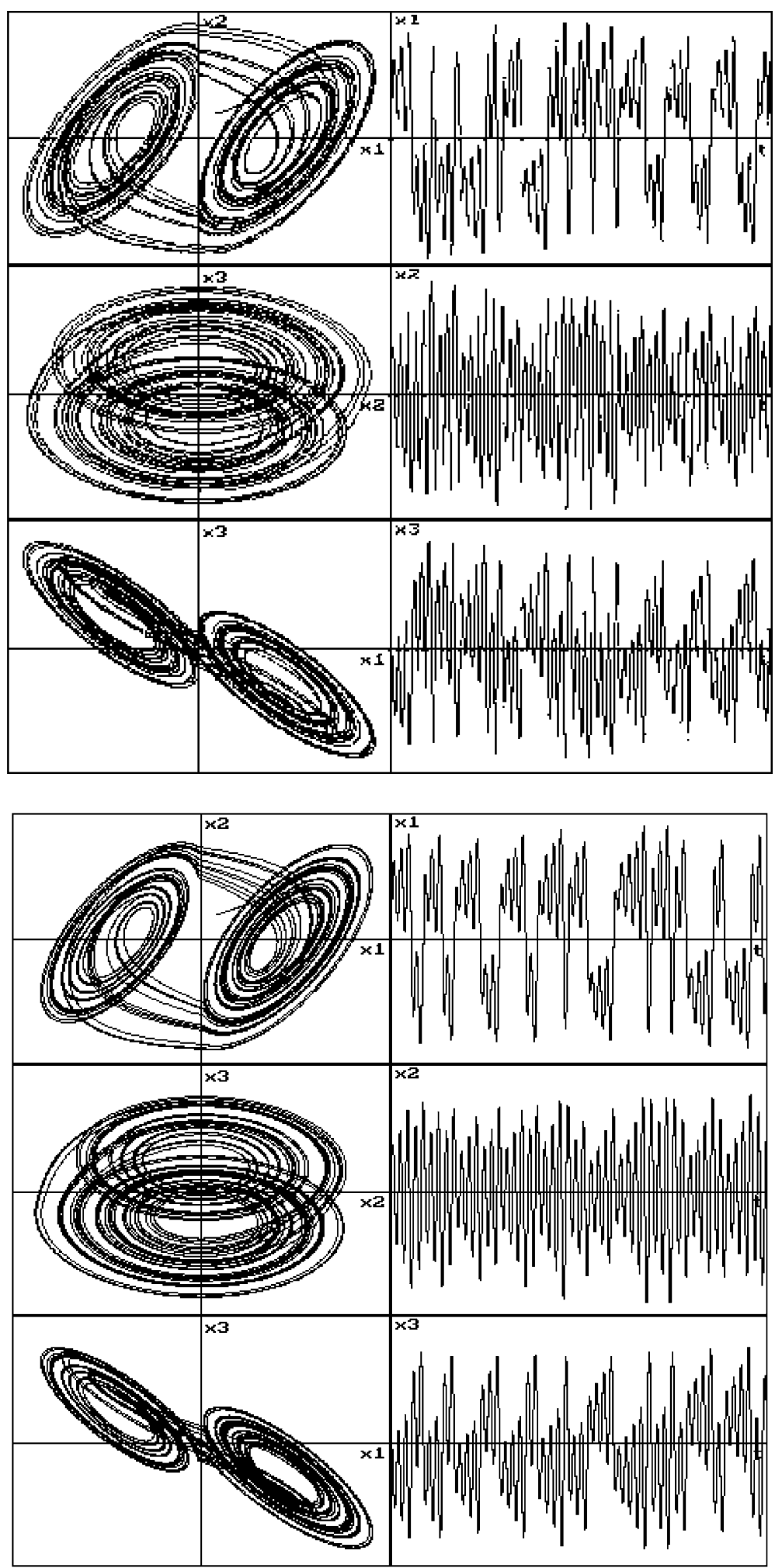

Fig. 7. (a) A chaotic trajectory of the system (8) with $\varepsilon=0.05$. (b) Another chaotic trajectory of the system (8) with $\varepsilon=0.1$. (c) A chaotic trajectory of differential inclusion (7) obtained with explicit Euler method for differential inclusions. 
(c)

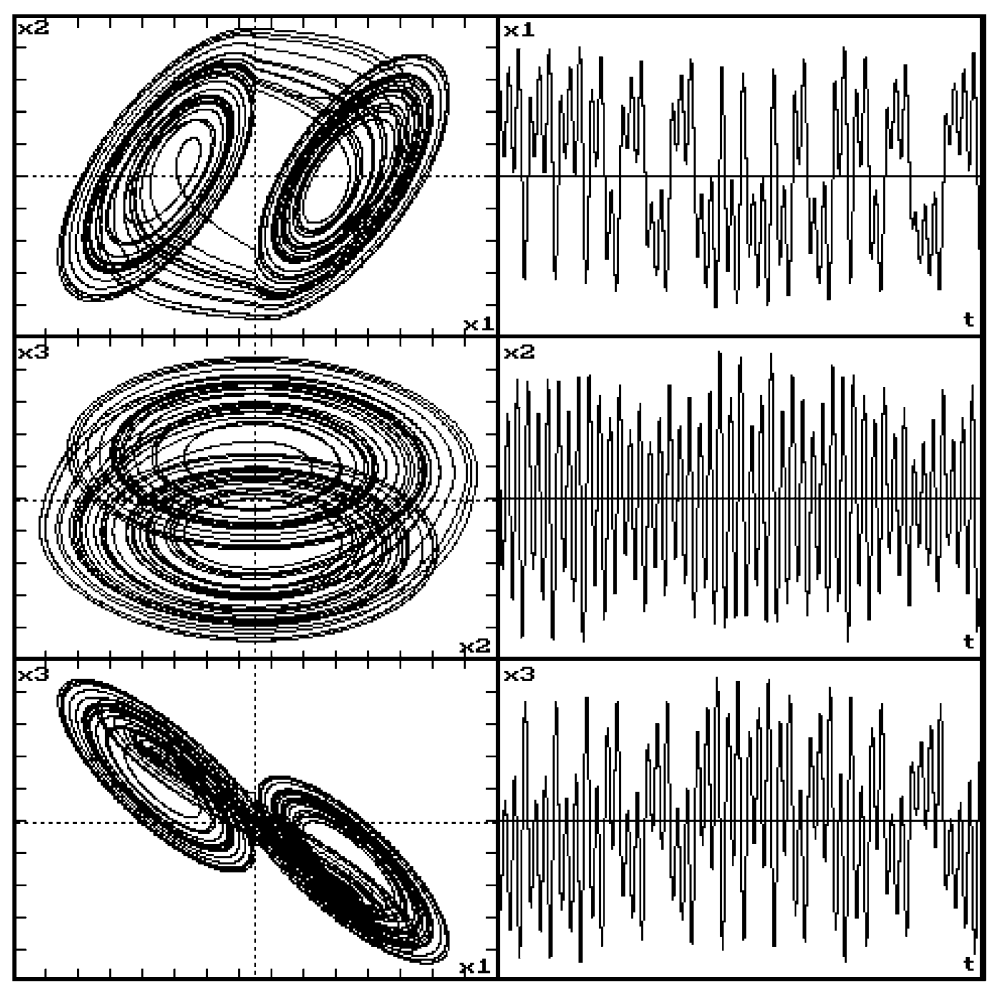

Fig. 7. (Continued).

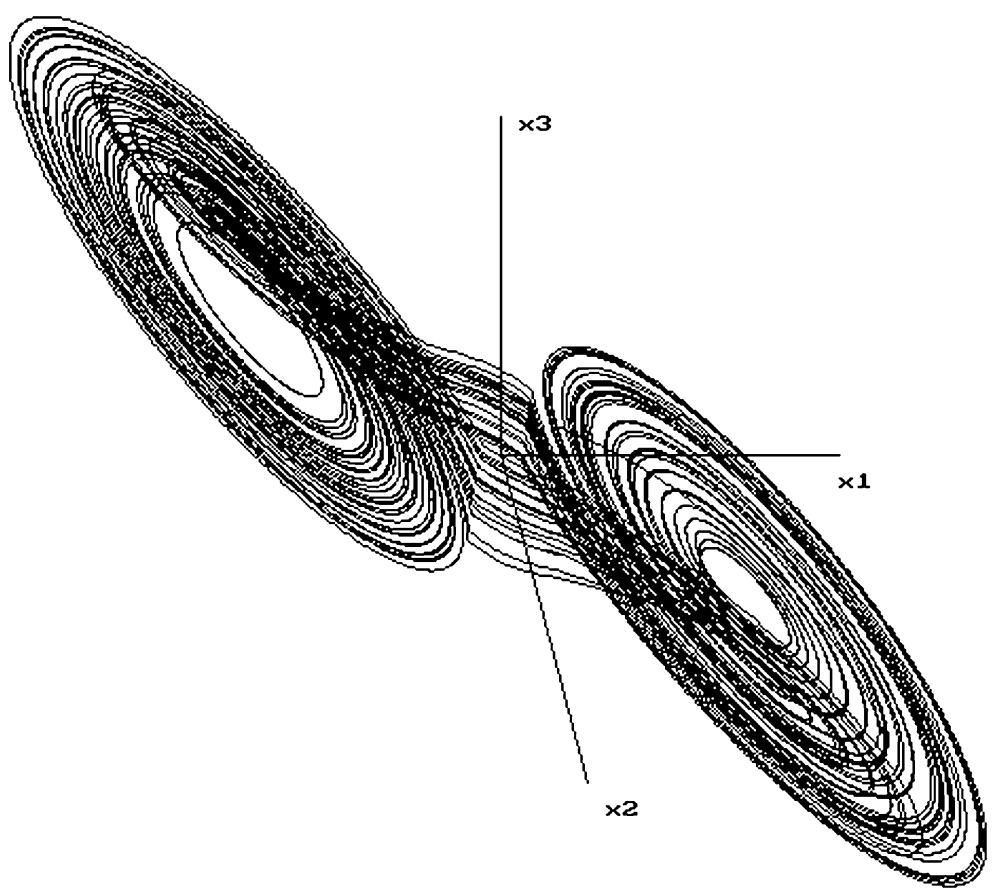

Fig. 8. A three-dimensional representation of a trajectory of the system (8). 


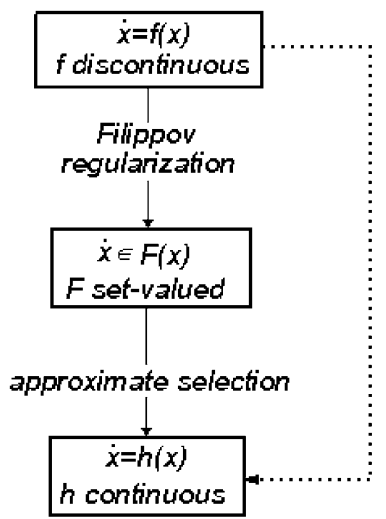

Fig. 9. Algorithm of the method.

\section{Appendix A}

Let us consider the metric spaces $\mathbf{R}^{n}$ and $\mathbf{R}$ with the attached Euclidean metric.

Definition 1. A subset $K \subset \mathbf{R}^{n}$ is called convex if for any $x, y \in K$ and $\lambda \in[0,1]$ one has $\lambda x+(1-\lambda) y \in K$. The convex hull of $K$ is the smallest convex set containing $K$ (the intersection of all convex sets containing $K)$.

Definition 2. Let $F: \Omega \subset \mathbf{R}^{n} \Rightarrow \mathbf{R}^{n}$ be a set-valued function. Its graph, $\operatorname{Graph}(F)$, is the subset of the product space $\Omega \times R^{n}$ defined by

$$
\operatorname{Graph}(F):=\left\{(x, y) \in \Omega \times \mathbf{R}^{n} \mid y \in F(x)\right\} .
$$

Filippov regularization. The Filippov regularization of a discontinuous function $f$ from an open subset $\Omega$ of $\mathbf{R}^{n}$ into $\mathbf{R}^{n}$, proposed by Filippov in [4], consists in replacing the function $f$ by

$$
F(\bar{x})=\operatorname{co} \operatorname{Lim}_{x \rightarrow \bar{x}} f(x), \quad x \in \Omega \backslash M, \quad \bar{x} \in \Omega,
$$

where co means the convex hull and the limit is the set of the limits of all convergent sequences $f\left(x_{k}\right)$ with $x_{k} \rightarrow x, M$ being the null set of the discontinuity points.

Definition 3. A single-valued function $x:[a, b] \subset \mathbf{R} \rightarrow \mathbf{R}^{n}$ is called absolutely continuous if $\forall \varepsilon>0, \exists \delta$ such that for any countable disjoint subintervals $\left[a_{k}, b_{k}\right]$ of $[a, b]$ such that

$$
\sum\left(b_{k}-a_{k}\right)<\delta
$$

we have

$$
\sum\left|x\left(b_{k}\right)-x\left(a_{k}\right)\right|<\varepsilon .
$$

Remark 1. An absolutely continuous function is at once continuous.

Definition 4. A single-valued function $g: \Omega \rightarrow \mathbf{R}^{n}$ is called a selection of a set-valued function $F$, if $g$ satisfies $g(x) \in F(x), \forall x \in \Omega$ (compare [7] and [8]).

Theorem 1 (Cellina approximate selection theorem [7]). Let $F$ be an upper semicontinuous function (see Definition 5) from $\Omega$ into the nonempty closed and convex subsets of $\mathbf{R}^{n}, F: \Omega \Rightarrow \mathbf{R}^{n}$. Then for every $\varepsilon>0$ there exists a locally Lipschitzean single-valued function $g: \Omega \rightarrow \mathbf{R}^{n}$ (see Definition 6 ) such that 


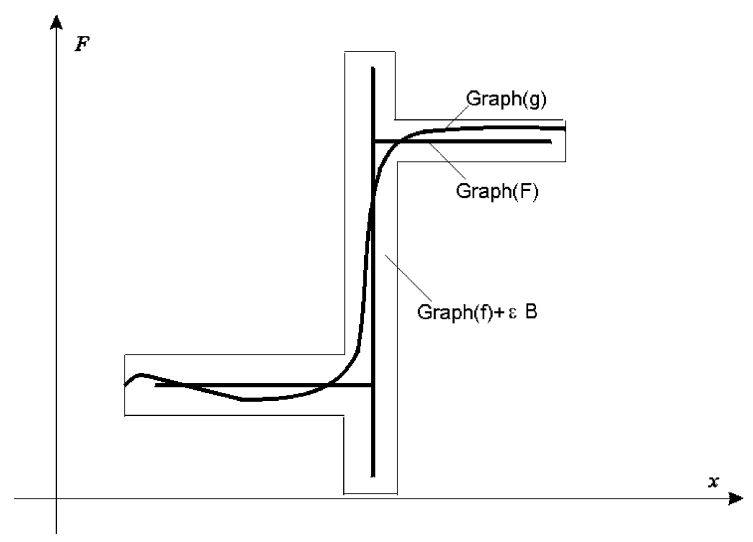

Fig. 10. Approximate selection $g$ of a set-valued function $F$.

$$
\operatorname{Graph}(g) \subset \operatorname{Graph}(F)+\varepsilon B,
$$

where $B$ is the n-dimensional unit ball centered in a point of Graph $(F)$ (see Fig. 10).

Definition 5. A set-valued function $F$ is called upper semicontinuous at $x \in \Omega$ if and only if for any neighborhood $U$ of $F(x)$ there exists a neighborhood $V$ of $x^{0}$, such that $F(V) \subset U$.

$F$ is upper semicontinuous if it is so at every $x \in \Omega$.

The function Sgn is upper semicontinuous.

Remark 2. The set-valued functions $F$ obtained from the functions $f \in \mathscr{F}$ by Filippov regularization are upper semicontinuous and verify the assumptions in Theorem 1.

Definition 6. A single-valued function $f$ is locally Lipschitzean if for any $x^{0} \in \mathbf{R}^{n}$ there exists a neighborhood of $x^{0}, N\left(x^{0}\right) \subset \mathbf{R}^{n}$ and a constant $L \geqslant 0$ (the Lipschitz constant) such that

$$
\forall x_{1}, x_{2} \in N\left(x^{0}\right),\left\|f\left(x_{2}\right)-f\left(x_{1}\right)\right\|<L\left\|x_{2}-x_{1}\right\|,
$$

where $\|\cdot\|$ is the Euclidean norm in $\mathbf{R}^{n}$.

Remark 3. Any Lipschitzean function is absolutely continuous, hence continuous (see Remark 1).

\section{References}

[1] Redheffer R, Port D. Differential equations, theory and applications. Boston: Jones and Bartlett; 1991.

[2] Dontchev A, Lempio F. Difference methods for differential inclusions: a survey. SIAM Rev 1992;34(2):261-94.

[3] Lempio F, Veliov V. Discrete approximation of differential inclusions. Bayreuter Math Schr 1998;54:149-232.

[4] Filippov AF. Differential equations with discontinuous righthand sides. Dordrecht: Kluwer Academic Publishers; 1988.

[5] Maresch AK. Implicit Runge-Kutta methods for differential inclusions. Numer Funct Anal Optimiz 1990-1991;11(9-10):937-58.

[6] Taubert K. Converging multistep methods for initial value problems involving multivalued maps. Computing 1981;27:123-36.

[7] Aubin J-P, Cellina A. Differential inclusions set-valued maps and viability theory. Berlin: Springer; 1984.

[8] Aubin J-P, Frankowska H. Set-valued analysis. Boston: Birkhäuser; 1990.

[9] Brown R. Generalizations of the Chua equations. IEEE Trans Circuits Syst Fundam Theory Applic 1993;40(11):878-83.

[10] Wiercigroch M, de Kraker B, Applied nonlinear dynamics and chaos of mechanical systems with discontinuities. Singapore: World Scientific; 2000. 